\title{
Association between sleep quality and cardiovascular damage in pre-dialysis patients with chronic kidney disease
}

Jun Zhang ${ }^{1 \dagger}$, Cheng Wang ${ }^{1 \dagger}$, Wenyu Gong ${ }^{1}$, Hui Peng ${ }^{1}$, Ying Tang ${ }^{2}$, Cui Cui Li ${ }^{1}$, Wenbo Zhao ${ }^{1}$, Zengchun Ye and Tanqi Lou ${ }^{1 *}$

\begin{abstract}
Background: Poor sleep quality, a novel risk factor of cardiovascular diseases (CVD), is highly prevalent in patients with chronic kidney disease (CKD). The association between poor sleep quality and cardiovascular damage in patients with CKD is unclear. This study is aimed to assess the prevalence and related risk factors of sleep disturbance and determine the relationship between sleep quality and cardiovascular damage in Chinese patients with pre-dialysis CKD.

Methods: A total of 427 pre-dialysis CKD patients (mean age $=39 \pm 15$ years, 260 male/167 female) were recruited in this study. The demographics and clinical correlates were collected. The sleep quality was measured by the Pittsburgh Sleep Quality Index (PSQI), whereas the cardiovascular damage indicators (the Early/late diastolic peak flow velocity (E/A) ratio and left ventricular mass index (LVMI)) were determined by an echocardiographic examination.

Results: Of the CKD patients, 77.8\% were poor sleepers as defined by a PSQI score $>5$. Median estimated glomerular filtration rate (eGFR) was 69.4(15.8-110.9) $\mathrm{ml} / \mathrm{min} / 1.73 \mathrm{~m}^{2}$. Logistic regression analysis revealed that left ventricular hypertrophy $(\mathrm{LVH})$ was independently associated with the PSQI score $(\mathrm{OR}=1.092,95 \% \mathrm{Cl}=1.011-1.179$, $p=0.025)$, after adjustment for age, sex and clinical systolic blood pressure, diastolic blood pressure, Phosphate, Intact parathyroid hormone (iPTH), Hemoglobin and eGFR. The linear regression analysis showed that the E/A ratios were independently associated with the PSQI score $(\beta=-0.115, P=0.028)$ after adjustment for a series of potential confounding factors.

Conclusions: Poor sleep quality, which is commonly found in pre-dialysis CKD patients, is an independent factor associated with cardiovascular damage in CKD patients. Our finding implies that the association between poor sleep and CVD might be mediated by cardiac remodeling.
\end{abstract}

Keywords: Sleep quality, Pittsburgh Sleep Quality Index (PSQI), Chronic kidney disease, Cardiovascular damage, Left ventricular hypertrophy (LVH)

\footnotetext{
*Correspondence: lou.tq@163.com

†Equal contributors

'Division of Nephrology, Department of Medicine, 3rd Affiliated Hospital of

Sun Yat-sen University, Guangzhou 510630, Guangdong, China

Full list of author information is available at the end of the article
} 


\section{Background}

Sleep-related problems and disorders are common problems in end-stage renal disease (ESRD) patients [1]. Several studies have shown that the prevalence of sleep problems including sleep apnea, insomnia, periodic limb movement disorder (PLMD), restless leg syndrome and overall poor sleep quality ranges from 30 to $80 \%$ among these patients $[1,2]$. There is substantial evidence indicating that insufficient sleep and poor sleep quality promote the development and exacerbate the severity of three important risk factors for chronic kidney disease (CKD), namely, type 2 diabetes, hypertension and obesity [3]. Poor sleep quality has been associated with an increased risk of mortality in pre-ESRD patients [4]. Recent studies have shown that poor sleep quality was related to the progression of cardiovascular disease (CVD) $[5,6]$. CVD is the most common cause of death in CKD patients in almost all stages. A previous study found that poor sleep quality was associated with a higher rate of CVD in continuous ambulatory peritoneal dialysis (CAPD) patients [7]. The pathophysiological pathways between poor sleep patterns and CVD remain unclear. Relatively few studies have examined sleep problems in the considerably larger group of patients with pre-dialysis CKD, and the related factors of poor sleep quality are not clear in these populations. Recent studies reported that the prevalence of poor sleep in CKD (prior to ESRD) varies widely from $14 \%$ to as high as $85 \%$ [3]. The association between sleep quality and specific laboratory variables was inconsistent in these studies. Kurella et al. reported a significant association between eGFR and sleep quality [8], whereas other studies failed to show a relationship between renal function and sleep quality $[4,9]$. Further research with a larger sample size and a different study population is needed.

There is no sleep quality data on Chinese pre-dialysis CKD patients. In this study, we aimed to assess the prevalence and associated risk factors of self-reported poor sleep quality and whether self-reported poor sleep quality is associated with biomarkers of cardiovascular damage (LVH and E/A ratio) disease in Chinese CKD patients.

\section{Methods}

The study protocol was approved by the Ethics Committee of the $3^{\text {rd }}$ hospital of Sun Yat-Sen University, and all of the study participants provided written informed consent before enrolling in this study.

\section{Patients}

The patients were recruited from May 2009 to Dec 2012. The CKD patients were diagnosed using the clinical practice guidelines set by the National Kidney Foundation Disease Outcomes Quality Initiative (NKF-K/DOQI) [10]. The inclusion criteria were as follows: 1 ) Pre-dialysis CKD patients with a stable disease state; 2) respondent to the sleep questionnaire and able to read and write and without a communication barrier. The exclusion criteria were as follows: 1 ) age $<18$ years; 2 ) treatment with corticosteroids or hormones; 3 ) acute changes in the estimated glomerular filtration rate (eGFR) $>30 \%$ in the previous 3 months; 4) pregnancy; 5) a history of abuse of drugs or alcohol; 6) night or shift work employment; 7) diagnosed sleep apnea and chronic obstructive pulmonary disease; 8) known acquired immunodeficiency syndrome (AIDS) or active infection; 9) acute cardiovascular disorders (unstable angina pectoris, heart failure, life-threatening arrhythmia, atrial fibrillation); 10) renal replacement therapy (hemodialysis, peritoneal dialysis or renal transplant); Body mass index more than $30 \mathrm{~kg} / \mathrm{m}^{2}$.

A total of 498 patients were diagnosed with CKD: 10 patients were treated with corticosteroids or hormones, 5 patients were $<18$ years old, 6 patients had an acute eGFR decrease, 8 patients had acute cardiovascular disorders, 9 patients had an infection, 12 patients were in renal replacement therapy and 3 patients had shift work employment, and these patients were excluded according to the exclusion criteria. Remaining patients $(n=445)$ was recruited for the study: 18 patients could not complete the questionnaire (10 patients could not read and write or had a communication barrier; 5 patients' questionnaires were invalid; and 3 patients refused to complete the questionnaire). Finally, 427 patients completed the questionnaire and were enrolled in the study.

\section{Measurement}

Sleep quality was measured using the Pittsburgh Sleep Quality Index (PSQI) [11], which contains 19 items. The PSQI is a self-administered questionnaire to assess a patient's sleep quality during the past month. From the patients' answers, the 19 questions are grouped into seven components scores, including subjective sleep quality, sleep latency, sleep duration, sleep efficiency, sleep disturbance, use of sleep medications, and daytime dysfunction, were calculated. Each component was scored from 0 to 3 to yield a global PSQI score from 0 to 21 . A high PSQI score indicates poor sleep quality. According to Buysse et al., patients with a PSQI score $>5$ are conventionally defined as "poor sleepers", whereas those with a score $\leq 5$ are considered "good sleepers". A previous study of primary insomniacs and healthy controls in community-dwelling adults reported that the cutoff score of 5 in the Chinese version PSQI has a sensitivity and specificity of $98 \%$ and $55 \%$, respectively [12].

Depression were assessed using the short form of the Beck Depression Inventory (BDI-SF) [13], a widely used 13-item questionnaire scored on a 4-point scale, from 0 to 3 , with overall scores ranging from 0 to 39 . Overall scores of 0-4 indicate no depression, 5-7 indicate mild, 
8-15 indicate moderate and 16 and more indicate severe depression. The BDI-SF has been found to have a good correlation with the standard 21-item BDI $(r=0.96$, $\mathrm{p}=0.001)$ and relates to the clinical depth-of-depression $(r=0.61)$ [13], with similar diagnostic efficiency [14]. We administered the Chinese version of the Beck Depression Inventory (BDI) to evaluate depression in the patients [15].

\section{Other data collected}

The clinical data recorded included duration of CKD, gender, age, current tobacco and alcohol intake, height and weight, blood pressure (BP), 24-h urinary protein excretion, serum albumin (ALB), serum creatinine (SCR), Serum uric acid, total cholesterol (TC), triglycerides (TG) and hemoglobin (HGB). The eGFR was estimated using the Chinese abbreviated MDRD (Modification of Diet in Renal Disease) equation [16]. The 24-h urinary protein excretion was calculated from a $24 \mathrm{~h}$ urine collection. The Scr, TC, ALB and TG concentrations were measured by a Hitachi 7180 biochemistry autoanalyzer (Hitachi, Tokyo, Japan; reagents from Roche Diagnostics, Mannheim, Germany). The Scr levels were measured by enzymatic and traceable to the isotope dilution mass spectrometry at the time of the study.

\section{Carotid ultrasonography}

Carotid intima-media thickness (cIMT) was assessed by two trained investigators before study commencement. A SonoSite MicroMaxx Ultrasound System paired with a 5-10-MHz Multifrequency High-resolution linear transducer (Bothell, WA, USA) with Sono-Calc IMT software was used for taking automatic measurements of cIMT. This was achieved by averaging three measurements taken on each carotid artery (anterior, lateral and posterior directions) and measuring the distance between the leading edge of the lumen-intima interface and the leading edge of the collagenous upper layer of the adventitia using high-resolution B-mode ultrasonography.

\section{Cardiac assessment}

Two-dimensional echocardiography was used to assess the volume, mass, systolic function and diastolic function of the left ventricle. The left ventricular mass was calculated using the Devereux method [17]. The left ventricular mass index (LVMI) was obtained by calculating the left ventricular mass to the height ${ }^{2.7}$ [18]. The males with a LVMI of $>49 \mathrm{~g} / \mathrm{m}^{2.7}$ and the females with a LVMI of $>45 \mathrm{~g} / \mathrm{m}^{2.7}$ were defined as having left ventricular hypertrophy (LVH) [19]. The left ventricular systolic function was assessed by the left ventricular ejection fraction (LVEF). Diastolic function was assessed by recording mitral flow with standard pulsed Doppler technique [20], and measurements of early diastolic peak flow velocity (E), late diastolic peak flow velocity $(\mathrm{A})$ and the ratio of early to late flow velocity peaks (E/A ratio).

\section{Statistical analyses}

The data were analyzed using SPSS software, version 15.0 (SPSS, Chicago, IL, USA). The descriptive statistics are presented as percentages for the discrete variables and as the means (standard deviation) for the continuous variables. The non-parametric variables are expressed as the median and interquartile range. The comparisons of the continuous variables between the groups were tested by Student's t-test or the non-parametric test. The differences among the categorical variables were analyzed using the $\chi^{2}$ test or the two-tailed Fisher's exact test, as appropriate.

In the univariate analyses, a linear regression model was employed to examine the association between the sleep quality scores (the dependent variable) and the independent variables, including duration of CKD, diabetes (yes/ no), current smoker (yes/no), alcohol intake (yes/no), BMI, BDI-SF score, clinic BP and clinical variables. A multiple linear regression (stepwise method) was performed to evaluate the relationship between the sleep quality scores and those independent variables with a potential association (crude $\mathrm{p}<0.15$ in univariate analyses) with a dependent variable in a univariate linear regression.

Univariate and multivariate logistic regression analyses were used to explore the independent associations of LVH (LVH vs. no LVH) with sleep quality scores. A univariate and multiple linear regression model was also employed to examine the association between the E/A ratio (dependent variable) and the sleep quality scores. All potential association variables (crude $\mathrm{p}<0.15$ in univariate analyses) and BDI score were tested further in multivariate regression analyses. All of the values are two-tailed, and the significance is defined as $\mathrm{p}<0.05$. Age and sex were forced to be included in the regression models for all of the regression analyses.

\section{Results}

\section{Sample characteristics}

The mean age of the subjects was $39 \pm 15$ years (range 18-70 years). Median estimated glomerular filtration rate (eGFR) was $69.4(15.8-110.9) \mathrm{ml} / \mathrm{min} / 1.73 \mathrm{~m}^{2}$. The study included 260 males (60.9\%) and 167 females (39.1\%). Of the patients, 44 (10.3\%) had diabetes; 186 patients (43.6\%) had hypertension. The average global PSQI score was $9.4 \pm 2.5$. A total of 332 patients $(77.8 \%)$ had poor sleep quality as defined as a PSQI score $>5$.

\section{Comparison of the clinical and laboratory data between the poor sleep quality group and the good sleep quality group}

Compared with the good sleepers, the patients with poor sleep quality had an increased LVMI level (47.1 \pm 
Table 1 Characteristics of the patients in the good sleepers group and the poor sleepers group

\begin{tabular}{|c|c|c|c|c|}
\hline Characteristic & $\begin{array}{l}\text { Overall } \\
(n=427)\end{array}$ & $\begin{array}{c}\text { Good sleepers } \\
\quad(n=95)\end{array}$ & $\begin{array}{l}\text { Poor sleepers } \\
\quad(n=332)\end{array}$ & $P$ value \\
\hline Age (years) & $39 \pm 15$ & $38 \pm 13$ & $39 \pm 15$ & 0.141 \\
\hline Sex ratio (M/F) & $260 / 167$ & $56 / 39$ & $204 / 128$ & 0.401 \\
\hline Duration of CKD (months) & $5(1-24)$ & $6(1-24)$ & $5(1-24)$ & 0.761 \\
\hline Current smoker, n (\%) & 79 (18.5\%) & $20(21.1 \%)$ & $59(17.8 \%)$ & 0.457 \\
\hline Alcohol intake, n (\%) & $26(6.1 \%)$ & $7(7.4 \%)$ & 19 (5.7\%) & 0.626 \\
\hline BMI $\left(\mathrm{kg} / \mathrm{m}^{2}\right)$ & $22.8 \pm 3.6$ & $22.7 \pm 4.0$ & $22.8 \pm 3.7$ & 0.784 \\
\hline ACEI/ARB (\%) & $240(56.2)$ & $56(58.9)$ & $184(55.4)$ & 0.560 \\
\hline Diabetes (\%) & $44(10.3)$ & $6(6.3)$ & $39(11.7)$ & 0.087 \\
\hline Proteinuria (g/24 h) & $1.41(0.46-3.82)$ & $1.40(0.46-4.12)$ & $1.42(0.46-3.76)$ & 0.793 \\
\hline Hemoglobin (g/L) & $118 \pm 29$ & $122 \pm 25$ & $117 \pm 29$ & 0.261 \\
\hline Albumin $(g / L)$ & $33.3 \pm 8.9$ & $33.0 \pm 9.0$ & $33.3 \pm 8.9$ & 0.810 \\
\hline Globulin( g/L) & $23.5 \pm 5.1$ & $23.2 \pm 5.2$ & $23.5 \pm 5.0$ & 0.713 \\
\hline Serum cystatin C (mg/L) & $1.5(1.0-3.5)$ & $1.4(1.0-2.5)$ & $1.4(0.9-3.4)$ & 0.450 \\
\hline Serum creatinine $(\mu \mathrm{mol} / \mathrm{L})$ & $104(69-355)$ & $89(66-214)$ & $114(70-423)$ & 0.024 \\
\hline eGFR $\left(\mathrm{ml} / \mathrm{min} / 1.73 \mathrm{~m}^{2}\right)$ & $69.4(15.8-110.9)$ & $85.3(32.3-121.2)$ & $65.9(13.2-109.6)$ & 0.040 \\
\hline Cholesterol (mmol/L) & $5.0(4.0-6.8)$ & $5.2(4.2-6.6)$ & $5.0(4.0-7.1)$ & 0.596 \\
\hline Triglycerides (mmol/L) & $1.7(1.1-2.6)$ & $1.8(1.1-2.9)$ & $1.8(1.1-2.7)$ & 0.513 \\
\hline LDL-C (mmol/L) & $3.1(2.3-4.5)$ & $3.2(2.6-4.3)$ & $3.1(2.3-4.6)$ & 0.426 \\
\hline $\mathrm{HDL}-\mathrm{C}(\mathrm{mmol} / \mathrm{L})$ & $1.3 \pm 0.5$ & $1.2 \pm 0.4$ & $1.3 \pm 0.5$ & 0.932 \\
\hline Glucose (mmol/L) & $4.7(4.2-5.3)$ & $4.6(4.2-5.0)$ & $4.7(4.2-5.3)$ & 0.391 \\
\hline Calcium (mg/dL) & $9.2 \pm 0.7$ & $9.1 \pm 0.7$ & $9.2 \pm 0.7$ & 0.139 \\
\hline Phosphate (mmol/L) & $1.4 \pm 0.5$ & $1.3 \pm 0.4$ & $1.4 \pm 0.5$ & 0.274 \\
\hline iPTH (pg/mL) & $54.6(32.1-150.2)$ & $48.1(32.0-83.2)$ & $54 . .7(31.1-153.0)$ & 0.173 \\
\hline Serum uric acid (umol/L) & $440 \pm 142$ & $441 \pm 139$ & $441 \pm 143$ & 0.967 \\
\hline LVEF (\%) & $66.9 \pm 7.7$ & $67.9 \pm 6.7$ & $66.7 \pm 7.9$ & 0.330 \\
\hline LVMI $\left(\mathrm{g} / \mathrm{m}^{2.7}\right)$ & $46.2 \pm 14.0$ & $41.7 \pm 9.5$ & $47.1 \pm 14.6$ & 0.019 \\
\hline LVH (\%) & $158(40.2)$ & $18(28.1)$ & $140(41.3)$ & 0.011 \\
\hline E/A ratio & $1.2 \pm 0.4$ & $1.3 \pm 0.5$ & $1.2 \pm 0.4$ & 0.027 \\
\hline CIMT (mm) & $0.66 \pm 0.20$ & $0.64 \pm 0.19$ & $0.67 \pm 0.20$ & 0.443 \\
\hline Hypertension (\%) & $186(43.6)$ & $43(45.3)$ & $143(43.1)$ & 0.813 \\
\hline $\mathrm{SBP}(\mathrm{mmHg})$ & $138.2 \pm 22.2$ & $137.9 \pm 20.9$ & $138.3 \pm 22.5$ & 0.921 \\
\hline $\mathrm{DBP}(\mathrm{mmHg})$ & $84.8 \pm 13.1$ & $85.1 \pm 11.0$ & $84.7 \pm 13.5$ & 0.826 \\
\hline Depression (\%) & $176(41.2)$ & $12(12.6)$ & $164(49.4)$ & $<0.001$ \\
\hline BDI-SF score & $3(1-7)$ & $1(0-2)$ & $4(2-8)$ & $<0.001$ \\
\hline PSQI score & $9.4 \pm 2.5$ & $3.5 \pm 1.3$ & $10.9 \pm 2.7$ & $<0.001$ \\
\hline \multicolumn{5}{|l|}{ CKD stage } \\
\hline CKD(I- II) (\%) & 222 & $55(24.8)$ & $167(75.2)$ & 0.117 \\
\hline CKD (III- V) (\%) & 205 & $40(19.5)$ & $165(80.5)$ & \\
\hline
\end{tabular}

(SBP: systolic blood pressure; DBP: diastolic blood pressure; BMI: body mass index, eGFR: estimated glomerular filtration rate; LDL-C: low-density lipoprotein cholesterol; HDL-C: high-density lipoprotein cholesterol; iPTH: intact parathyroid hormone; SBP: systolic blood pressure; DBP: diastolic blood pressure; BDI-SF: short form of the Beck Depression Inventory; CKD: Chronic kidney disease; CIMT: Carotid intima-media thickness; E/A ratio: Early/late diastolic peak flow velocity ratio; LVH: Left ventricular hypertrophy; LVMI: left ventricular mass index; Serum calcium is corrected by the following formula:[calcium] (mg/dL) = measured [calcium] + ( 4.0-[serum albumin(mg/ dl)]) $\times 0.8$, PSQl: Pittsburgh Sleep Quality Index). 
Table 2 Univariate and multivariate Linear Regression model for predictors of sleep quality (PSQI scores)

\begin{tabular}{|c|c|c|c|c|}
\hline & $\begin{array}{c}\text { Standardized } \\
\text { coefficient } \beta\end{array}$ & $\begin{array}{l}\text { Crude } \\
\text { P value }\end{array}$ & $\begin{array}{c}\text { Adjusted } \\
\text { standardized } \\
\text { coefficient } \beta\end{array}$ & $\begin{array}{l}\text { Adjusted } \\
\text { P value }^{\mathrm{b}}\end{array}$ \\
\hline Age (years) & 0.149 & 0.004 & 0.155 & 0.004 \\
\hline Duration of CKD (months) & 0.104 & 0.054 & - & - \\
\hline Male vs. female & 0.024 & 0.637 & - & - \\
\hline Current smoker (no vs. yes) & -0.046 & 0.283 & & \\
\hline Alcohol intake (no vs. yes) & -0.039 & 0.368 & & \\
\hline $\mathrm{BMI}\left(\mathrm{kg} / \mathrm{m}^{2}\right)$ & 0.010 & 0.863 & & \\
\hline Diabetes (no vs. yes) & 0.086 & 0.116 & - & - \\
\hline Proteinuria (g/24 h) & -0.047 & 0.398 & & \\
\hline Hemoglobin (g/L) & -0.174 & 0.004 & - & - \\
\hline Albumin $(\mathrm{g} / \mathrm{L})$ & 0.065 & 0.224 & & \\
\hline eGFR (ml/min/1.73 $\left.\mathrm{m}^{2}\right)$ & -0.224 & $<0.001$ & - & - \\
\hline Cholesterol (mmol/L) & -0.031 & 0.575 & & \\
\hline Triglyceride $(\mathrm{mmol} / \mathrm{L})$ & -0.033 & 0.543 & & \\
\hline LDL-C (mmol/L) & -0.054 & 0.339 & & \\
\hline Calcium (mg/dL) & 0.073 & 0.152 & & \\
\hline Phosphate (mmol/L) & 0.140 & 0.007 & - & - \\
\hline iPTH (pg/mL) & 0.139 & 0.018 & - & - \\
\hline Serum uric acid (umol/L) & 0.029 & 0.587 & & \\
\hline $\mathrm{SBP}(\mathrm{mmHg})$ & 0.030 & 0.585 & & \\
\hline $\mathrm{DBP}(\mathrm{mmHg})$ & 0.064 & 0.218 & & \\
\hline BDI-SF score & 0.413 & $<0.001$ & 0.427 & $<0.001$ \\
\hline
\end{tabular}

( ${ }^{a}$ all of the variables were adjusted by age, sex. ${ }^{\mathrm{b}}$ The age, sex, duration of CKD, diabetic vs. non diabetic status, eGFR, hemoglobin, phosphate, iPTH and BDI-score were included in the multivariate model (stepwise method). -Without significance and not able to be included in the multivariate model. eGFR: estimated glomerular filtration rate; iPTH: intact parathyroid hormone, SBP: systolic blood pressure; DBP: diastolic blood pressure; BDI-SF: short form of the Beck Depression Inventory).

$14.6 \mathrm{~g} / \mathrm{m}^{2.7}$ vs. $\left.41.7 \pm 9.5 \mathrm{~g} / \mathrm{m}^{2.7}, \mathrm{P}<0.05\right)$, a higher proportion of LVH (41.3\% vs. 28.1\%; $p<0.05)$, a lower E/A ratio $(1.2 \pm 0.4$ vs. $1.3 \pm 0.5, \mathrm{P}<0.05)$, a higher $\mathrm{BDI}$ score (4 (2-8) vs. $1(0-2) ; \mathrm{p}<0.001)$. The poor sleep quality group had a higher level of serum creatinine [114 (70-423) umol/L vs. 89 (66-214) umol/L, $\mathrm{P}<0.05$ ] and a lower eGFR value $\left[65.9(13.2-109.6) \mathrm{ml} / \mathrm{min} / 1.73 \mathrm{~m}^{2}\right.$ VS. $\left.85.3(32.3-121.2) \mathrm{ml} / \mathrm{min} / 1.73 \mathrm{~m}^{2}, \mathrm{P}<0.05\right]$. The two subgroups were similar for the other variables. The results are shown in Table 1.

\section{Independent characteristics of patients with poor sleep quality}

Table 2 demonstrates the strengths of the associations between sleep quality and the clinical and laboratory characteristics. The univariate linear regression analysis revealed that older age, a longer duration of CKD, lower eGFR, lower hemoglobin level, higher BDI scores, higher serum levels of phosphate and iPTH were significantly associated with poor sleep quality. We found that only older age and higher BDI scores were independently associated with poor sleep quality in the multivariate regression analysis.

\section{Association of sleep quality with LVH}

Figure 1 shows that the LVH patients had higher PSQI scores when compared with the non-LVH group $(\mathrm{P}<0.001)$. Table 3 shows the associations of LVH and

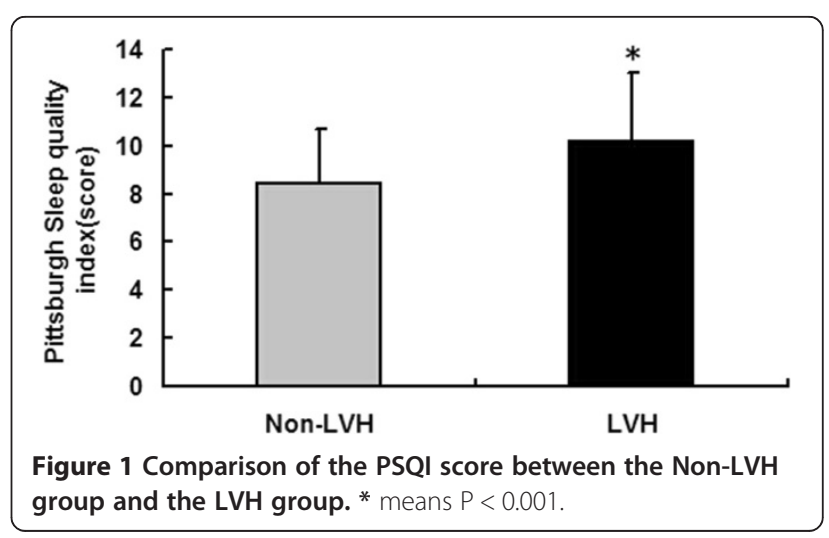


Table 3 Univariate and multivariate logistic regression analysis for left ventricular hypertrophy (1 = no LVH; 2 = LVH)

\begin{tabular}{|c|c|c|c|c|}
\hline & $\mathrm{OR}(95 \% \mathrm{Cl})$ & $\begin{array}{l}\text { Crude } \\
\text { P value }^{\mathrm{a}}\end{array}$ & $\begin{array}{l}\text { Adjusted } \\
\text { OR(95\% Cl) }\end{array}$ & $\begin{array}{l}\text { Adjusted } \\
\text { P value }^{b}\end{array}$ \\
\hline Age (per 1 year) & $1.061(1.040-1.082)$ & $<0.001$ & - & - \\
\hline Duration of CKD (per 1 month) & $0.999(0.993-1.005)$ & 0.759 & - & - \\
\hline Male vs. female & $1.525(0.879-2.645)$ & 0.133 & - & - \\
\hline Current smoker (no vs. yes) & $0.931(0.476-1.822)$ & 0.835 & & \\
\hline Alcohol intake (no vs. yes) & $1.980(0.780-5.027)$ & 0.151 & & \\
\hline BMI (per $\left.1 \mathrm{~kg} / \mathrm{m}^{2}\right)$ & $0.992(0.908-1.083)$ & 0.855 & & \\
\hline Diabetes (no vs. yes) & $1.203(0.614-2.989)$ & 0.690 & & \\
\hline ACEI/ARB (no/yes) & $0.768(0.431-1.368)$ & 0.370 & & \\
\hline Proteinuria (per $1 \mathrm{~g} / 24 \mathrm{~h}$ ) & $0.939(0.815-1.081)$ & 0.381 & & \\
\hline Hemoglobin (per $1 \mathrm{~g} / \mathrm{L}$ ) & $0.971(0.960-0.983)$ & $<0.001$ & - & - \\
\hline ALB (per $1 \mathrm{~g} / \mathrm{L}$ ) & $1.017(0.977-1.061)$ & 0.421 & & \\
\hline $\mathrm{eGFR}<60 \mathrm{ml} / \mathrm{min} / 1.73 \mathrm{~m}^{2}$ & $8.150(3.234-20.542)$ & $<0.001$ & $3.943(1.255-12.387)$ & 0.019 \\
\hline Cholesterol (per $1 \mathrm{mmol} / \mathrm{L}$ ) & $0.940(0.844-1.048)$ & 0.264 & & \\
\hline Triglyceride (per $1 \mathrm{mmol} / \mathrm{L}$ ) & $0.930(0.711-1.217)$ & 0.598 & & \\
\hline LDL-C (per $1 \mathrm{mmol} / \mathrm{L}$ ) & $0.936(0.816-1.047)$ & 0.347 & & \\
\hline Calcium (per 1 mg/dL) & $0.397(0.223-0.646)$ & $<0.001$ & - & - \\
\hline Phosphate (per 1 mg/dL) & $2.998(1.500-5.992)$ & 0.002 & - & - \\
\hline iPTH (per 1 pg/mL) & $1.006(1.003-1.010)$ & $<0.001$ & $1.004(1.002-1.007)$ & 0.001 \\
\hline Serum uric acid (per 1 umol/L) & $1.003(1.001-1.006)$ & 0.004 & - & - \\
\hline PSQI score & $1.117(1.041-1.200)$ & 0.002 & $1.092(1.011-1.179)$ & 0.025 \\
\hline BDI-SF score & $1.061(0.012-1.111)$ & 0.014 & - & - \\
\hline SBP (per $1 \mathrm{mmHg}$ ) & $1.044(1.028-1.061)$ & $<0.001$ & $1.034(1.015-1.054)$ & $<0.001$ \\
\hline DBP (per 1 mmHg) & $1.056(1.032-1.081)$ & $<0.001$ & - & - \\
\hline
\end{tabular}

( ${ }^{\text {a all }}$ of the variables were adjusted by age, sex. ${ }^{\text {b}}$ The age, sex, hemoglobin, eGFR, phosphate, serum calcium, Serum uric acid, iPTH, SBP, DBP, BDI -score and PSQI score were included in the multivariate model (backward method). -Without significance and not able to be included in the multivariate model. eGFR: the estimated glomerular filtration rate; iPTH: intact parathyroid hormone, SBP: systolic blood pressure; DBP: diastolic blood pressure; PSQI: Pittsburgh Sleep Quality Index).

the demographic and clinical factors. Age; the levels of hemoglobin, serum calcium, phosphate and iPTH; Serum uric acid; eGFR $<60 \mathrm{ml} / \mathrm{min} / 1.73 \mathrm{~m}^{2}$; SBP and DBP; and poor sleep quality were significantly associated with $\mathrm{LVH}$ when adjusted only for age and gender. In the multivariate regression analysis, an eGFR $<60 \mathrm{ml} / \mathrm{min} / 1.73 \mathrm{~m}^{2}$, a higher serum iPTH level, higher SBP and poor sleep quality were independently associated with LVH.

\section{Association of sleep quality with the E/A ratio}

Figure 2 shows that the PSQI score was significantly associated with the E/A ratio $(r=0.190, p<0.001)$ and was not associated with the CIMT $(\mathrm{r}=-0.021, \mathrm{p}=0.724)$. As shown in Table 4, the linear regression analyses revealed that age, the serum phosphate, BMI, Serum uric acid, SBP, DBP and PSQI scores were negatively associated with the E/A ratio, whereas the eGFR was positively associated with the E/A ratio when adjusted only with age and gender. We found that age, the DBP, BMI and PSQI scores were independent factors associated with the E/A ratio in the further multivariate regression analysis.

\section{Discussion}

Few studies have addressed sleep quality in subjects with CKD prior to ESRD. Our study first investigated sleep quality in Chinese CKD patients prior to dialysis and found that the prevalence of poor sleep quality was as high as $77.8 \%$. Previous studies have shown that the prevalence of sleep disorders based on questionnaires have provided widely different estimates of in-patients with CKD, which ranged from 14-85\% in CKD patients [3], 41-83\% in HD patients [21-23] and $49-85 \%$ in PD patients $[7,21,24]$. These rates might vary be because of the different life styles, genetic factors, environmental conditions and primary cause of CKD in the study populations or because different questionnaires were used to estimate sleep quality in the studies. Our study shows that a higher sleep quality score was positively associated 

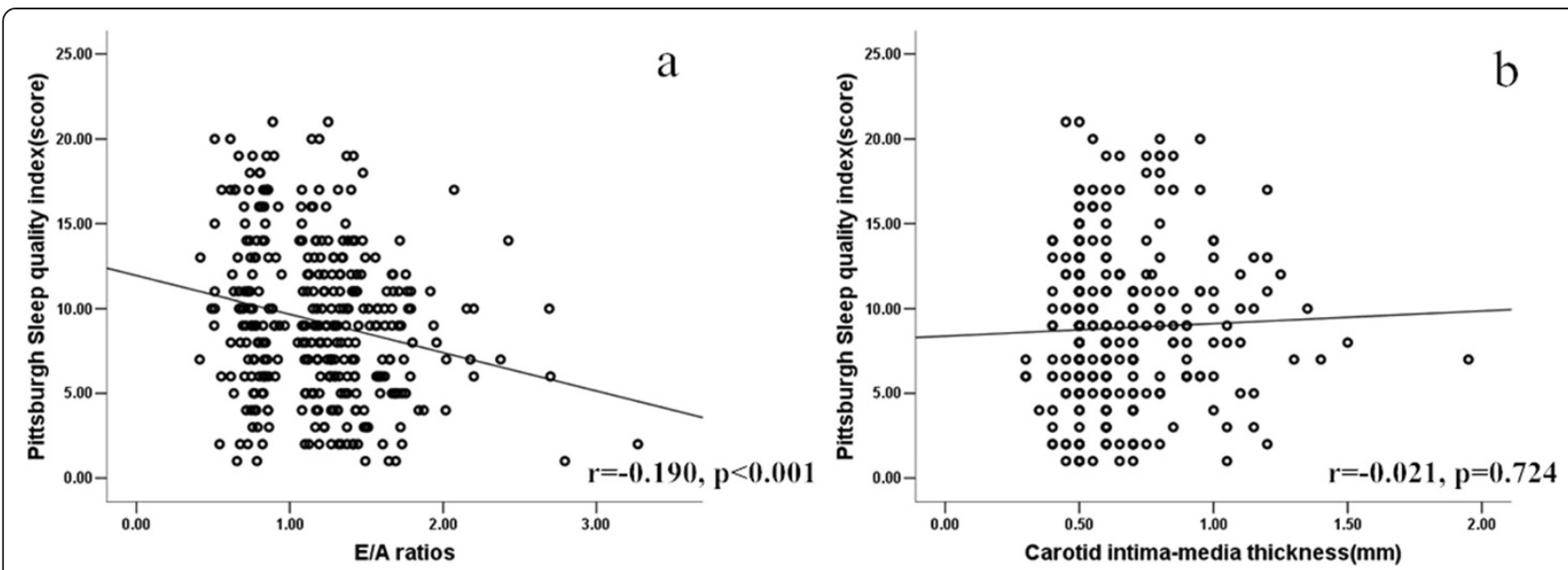

Figure 2 Correlation analysis between the PSQI score and the E/A ratios (a) and CIMT(b).

with older age, higher phosphate levels, $\mathrm{iPTH}$ and a longer duration of CKD and inversely associated with the hemoglobin level and the eGFR in the univariate analyses. After adjusting for the confounding factors, only higher BDI scores and older age were independently associated with sleep quality. Numerous factors could potentially contribute to the high prevalence of sleep problems in CKD patients including older age, pain, depression, dyspnea, nausea and pruritus; however, it appears that there is no association between sleep quality and specific laboratory variables such as hemoglobin, albumin, calcium or phosphorus in CKD patients [4,25]. As in most of the studies $[4,9,26]$, our study failed to show a relationship between renal function and sleep quality in the multivariate linear regression analysis, suggested that poor sleep quality could exist at a very early stage in CKD and that the decreased renal function and other specific laboratory variables might not be the direct cause of poor sleep in CKD patients. A series of associated comorbidities and symptoms such as high depression scores, pain, nausea and itchiness could be aggravated by declining renal function and affect the sleep quality in CKD patients [4]. In research with a small sample size $(n=58)$, Kurella et al. [8] reported a significant association between eGFR and sleep quality among non-African-American subjects.

Previous studies have shown that poor sleep quality is an important risk factor for the development of cardiovascular diseases [5] in the general population. The PSQI score was reported to be positively associated with cardiovascular disease in CAPD patients [7]. Recent research indicated that an increase in observed/predicted LVM is independently associated with adverse cardiovascular outcomes in patients with stages 3-5 CKD [27]. Diastolic dysfunction is a strong predictor of mortality in patients with CKD [28]. From the univariate and multivariate regression analysis, our study first showed that higher PSQI scores were associated with $\mathrm{LVH}$ and lower E/A ratios.
The mechanisms of these associations are unclear. One of the explanations might be that poor sleep quality is associated with blunting of the nocturnal dip in blood pressure and with higher activation of the sympathetic nervous system [29]; the sympathetic nervous system and nondipping blood pressure are risk factors of LVH and lower E/A ratios [30,31]. Reduced sleep quality might decrease the normal nocturnal increase in the plasma renin activity and aldosterone levels [3] and might lead to LVH. Our findings might facilitate a better understanding of the pathogenesis and consequences of cardiovascular diseases in CKD patients with poor sleep quality.

The strength of our study was that we were the first to report the prevalence and risk factors of poor sleep quality in Chinese pre-dialysis CKD patients; we first reported that poor sleep quality is closely related with cardiovascular damage. Several limitations should be considered when interpreting our findings. First, the subjective reporting of sleep quality by use of the PSQI might lead to reporting bias. It has been shown that patients with sleep problems are more likely to overestimate their sleep difficulties [32]. In addition, the PSQI measures overall general sleep quality rather than a specific form of sleep disorder. Future studies with objective measures are warranted, but using a polysomnogram or other objective measure is costly in a large clinical study such as this study. Second, the nature of the cross-sectional design in this study did not allow us to determine a causal relationship between poor sleep quality and cardiovascular damage and pre-dialysis patients. Cardiovascular damage might lead to poor sleep quality [33,34]. Further multi-center follow-up studies with larger samples are necessary to determine the effect of factors of such as poor sleep quality and to investigate the complex nature of the relationship between sleep quality and cardiovascular damage in Chinese CKD patients. In addition, although our study excluded sleep apnea and chronic obstructive pulmonary disease and find 
Table 4 Univariate and multivariate Linear Regression model for the predictors of the E/A ratios

\begin{tabular}{|c|c|c|c|c|}
\hline & & P value $^{a}$ & Coefficient $\beta$ & $\mathrm{P}$ value $^{\mathrm{b}}$ \\
\hline Age (years) & -0.650 & $<0.001$ & -0.613 & $<0.001$ \\
\hline Duration of CKD (months) & -0.059 & 0.186 & & \\
\hline Male vs. female & -0.032 & 0.459 & - & - \\
\hline Current smoker (no vs. yes) & -0.054 & 0.318 & & \\
\hline Alcohol intake (no vs. yes) & -0.024 & 0.585 & & \\
\hline $\mathrm{BMI}\left(\mathrm{kg} / \mathrm{m}^{2}\right)$ & -0.139 & 0.002 & -0.113 & 0.029 \\
\hline ACEI/ARB (no/yes) & 0.041 & 0.369 & & \\
\hline Diabetes (no vs. yes) & -0.060 & 0.192 & & \\
\hline Proteinuria (g/24 h) & -0.005 & 0.907 & & \\
\hline Hemoglobin (g/L) & 0.008 & 0.870 & & \\
\hline Albumin $(\mathrm{g} / \mathrm{L})$ & -0.031 & 0.485 & & \\
\hline eGFR $\left(\mathrm{ml} / \mathrm{min} / 1.73 \mathrm{~m}^{2}\right)$ & 0.126 & 0.012 & - & - \\
\hline Cholesterol (mmol/L) & -0.048 & 0.286 & & \\
\hline Triglyceride $(\mathrm{mmol} / \mathrm{L})$ & -0.060 & 0.168 & & \\
\hline LDL-C (mmol/L) & -0.053 & 0.247 & & \\
\hline Calcium (mg/dL) & -0.064 & 0.138 & - & - \\
\hline Phosphate (mmol/L) & -0.096 & 0.025 & - & - \\
\hline iPTH (pg/mL) & -0.068 & 0.140 & - & - \\
\hline Serum uric acid (umol/L) & -0.168 & $<0.001$ & - & - \\
\hline PSQI scores & -0.129 & 0.007 & -0.115 & 0.028 \\
\hline BDI-SF score & -0.065 & 0.229 & - & - \\
\hline $\mathrm{SBP}(\mathrm{mmHg})$ & -0.173 & $<0.001$ & - & - \\
\hline $\mathrm{DBP}(\mathrm{mmHg})$ & -0.191 & $<0.001$ & -0.184 & $<0.001$ \\
\hline
\end{tabular}

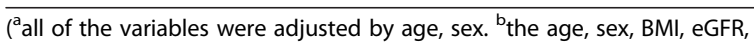
serum calcium, phosphate and iPTH, Serum uric acid, SBP, DBP, BDI score and PSQI scores were included in the multivariate model (stepwise method). -Without significance and not able to be included in the multivariate model and hence these independent variables were not included into the model to predict dependent variable. DBP: diastolic blood pressure; eGFR: estimated glomerular filtration rate; iPTH: intact parathyroid hormone, SBP: systolic blood pressure; PSQI: Pittsburgh Sleep Quality Index).

that depression can affect sleep quality, other factors, such as pruritis, chronic pain and caffeine use might affect sleep, were not include in this study. These may also confound the results. Finally, the studied population in this study was relatively young. These findings in the current study might not be able to generalize to older population.

\section{Conclusions}

This study demonstrates a high prevalence of poor sleep quality in pre-dialysis CKD patients. Poor sleep quality is an independent risk factor for LVH and ventricular diastolic function in pre-dialysis CKD patients. Assessment with the PSQI is the first step in detecting sleep disorders and should be used in all renal patients to determine for whom more accurate procedures are needed to identify and treat specific disorders. Treatment to improve sleep quality might improve the overall quality of life and positively affect CVD.

\section{Competing interests \\ No financial or other conflicts of interest are declared by the authors.}

\section{Authors' contributions}

Conceived and designed the experiments: JZ and TL. Performed the experiments: CW, WG, YT, CL, WZ and ZY. Analyzed the data: JZ and CW. Contributed reagents/materials/analysis tools: HP and ZY. Wrote the paper: JZ. Review the manuscript for intellectual content: Dr CW, JZ and TL. All authors read and approved the final manuscript.

\section{Acknowledgements}

We would like to thank all patients and their families for participating in this study.

This project was supported by the National Natural Science Foundation of China (No.81300595)

\section{Author details}

'Division of Nephrology, Department of Medicine, 3rd Affiliated Hospital of Sun Yat-sen University, Guangzhou 510630, Guangdong, China. ${ }^{2}$ Division of Nephrology, Department of Medicine, 2rd Affiliated Hospital of Sun Yat-sen University, Guangzhou 510120, Guangdong, China.

Received: 3 April 2014 Accepted: 5 August 2014

Published: 12 August 2014

References

1. Parker KP: Sleep disturbances in dialysis patients. Sleep Med Rev 2003, 7:131-143.

2. Unruh ML, Buysse DJ, Dew MA, Evans IV, Wu AW, Fink NE, Powe NR, Meyer KB: Sleep quality and its correlates in the first year of dialysis. Clin J Am Soc Nephrol 2006, 1:802-810.

3. Turek NF, Ricardo AC, Lash JP: Sleep disturbances as nontraditional risk factors for development and progression of CKD: review of the evidence. Am J Kidney Dis 2012, 60:823-833.

4. Kumar B, Tilea A, Gillespie BW, Zhang X, Kiser M, Eisele G, Finkelstein F, Kotanko P, Levin N, Rajagopalan S: Significance of self-reported sleep quality (SQ) in chronic kidney disease (CKD): the Renal Research Institute (RRI)-CKD study. Clin Nephrol 2010, 73:104-114.

5. Hoevenaar-Blom MP, Spijkerman AM, Kromhout D, van den Berg JF, Verschuren WM: Sleep duration and sleep quality in relation to 12-year cardiovascular disease incidence: the MORGEN study. Sleep 2011, 34:1487-1492.

6. Suzuki E, Yorifuji T, Ueshima K, Takao S, Sugiyama M, Ohta T, Ishikawa-Takata K, Doi H: Sleep duration, sleep quality and cardiovascular disease mortality among the elderly: a population-based cohort study. Prev Med 2009, 49:135-141.

7. Li J, Guo Q, Ye X, Lin J, Yi C, Mao H, Yang X, Yu X: Prevalence and risk factors of sleep disturbance in continuous ambulatory peritoneal dialysis patients in Guangzhou, southern China. Int Urol Nephrol 2012, 44:929-936.

8. Kurella M, Luan J, Lash JP, Chertow GM: Self-assessed sleep quality in chronic kidney disease. Int Urol Nephrol 2005, 37:159-165.

9. Sabbatini M, Pisani A, Crispo A, Ragosta A, Gallo R, Pota A, Serio V, Tripepi G, Cianciaruso B: Sleep quality in patients with chronic renal failure: a 3-year longitudinal study. Sleep Med 2008, 9:240-246.

10. $\bigotimes: \mathrm{K} / \mathrm{DOQ}$ clinical practice guidelines for chronic kidney disease: evaluation, classification, and stratification. Am J Kidney Dis 2002, 39(Suppl 1):1-266.

11. Buysse DJ, Reynolds CR, Monk TH, Berman SR, Kupfer DJ: The Pittsburgh sleep quality index: a new instrument for psychiatric practice and research. Psychiatry Res 1989, 28:193-213.

12. Tsai PS, Wang SY, Wang MY, Su CT, Yang TT, Huang CJ, Fang SC: Psychometric evaluation of the Chinese version of the Pittsburgh sleep quality index (CPSQI) in primary insomnia and control subjects. Qual Life Res 2005, 14:1943-1952.

13. Beck AT, Beck RW: Screening depressed patients in family practice. A rapid technic. Postgrad Med 1972, 52:81-85. 
14. Bennett DS, Ambrosini PJ, Bianchi M, Barnett D, Metz C, Rabinovich H: Relationship of beck depression inventory factors to depression among adolescents. J Affect Disord 1997, 45:127-134.

15. Min Z, Xu L, Chen H, Ding X, Yi Z, Mingyuang Z: A pilot assessment of relapse prevention for heroin addicts in a Chinese rehabilitation center. Am J Drug Alcohol Abuse 2011, 37:141-147.

16. Ma YC, Zuo L, Chen JH, Luo Q, Yu XQ, Li Y, Xu JS, Huang SM, Wang LN, Huang W: Modified glomerular filtration rate estimating equation for Chinese patients with chronic kidney disease. J Am Soc Nephrol 2006, 17:2937-2944.

17. Schiller NB, Shah PM, Crawford M, DeMaria A, Devereux R, Feigenbaum H, Gutgesell H, Reichek N, Sahn D, Schnittger I: Recommendations for quantitation of the left ventricle by two-dimensional echocardiography. American Society of Echocardiography Committee on Standards, Subcommittee on Quantitation of Two-Dimensional Echocardiograms. J Am Soc Echocardiogr 1989, 2:358-367.

18. Zoccali C, Benedetto FA, Mallamaci F, Tripepi G, Giacone G, Cataliotti A, Seminara G, Stancanelli B, Malatino LS: Prognostic impact of the indexation of left ventricular mass in patients undergoing dialysis. J Am Soc Nephrol 2001, 12:2768-2774.

19. Glassock RJ, Pecoits-Filho R, Barberato SH: Left ventricular mass in chronic kidney disease and ESRD. Clin J Am Soc Nephrol 2009, 4(Suppl 1):79-91.

20. Ommen SR, Nishimura RA: A clinical approach to the assessment of left ventricular diastolic function by Doppler echocardiography: update 2003. Heart 2003, 89(Suppl 3):18-23.

21. Eryavuz N, Yuksel S, Acarturk G, Uslan I, Demir S, Demir M, Sezer MT: Comparison of sleep quality between hemodialysis and peritoneal dialysis patients. Int Urol Nephrol 2008, 40:785-791.

22. Mucsi I, Molnar MZ, Ambrus C, Szeifert L, Kovacs AZ, Zoller R, Barotfi S, Remport A, Novak M: Restless legs syndrome, insomnia and quality of life in patients on maintenance dialysis. Nephrol Dial Transplant 2005, 20:571-577.

23. Elder SJ, Pisoni RL, Akizawa T, Fissell R, Andreucci VE, Fukuhara S, Kurokawa K, Rayner HC, Furniss AL, Port FK, et al: Sleep quality predicts quality of life and mortality risk in haemodialysis patients: results from the Dialysis Outcomes and Practice Patterns Study (DOPPS). Nephrol Dial Transplant 2008, 23:998-1004

24. Yang JY, Huang JW, Chiang CK, Pan CC, Wu KD, Tsai TJ, Chen WY: Higher plasma interleukin-18 levels associated with poor quality of sleep in peritoneal dialysis patients. Nephrol Dial Transplant 2007, 22:3606-3609.

25. Cohen SD, Patel SS, Khetpal P, Peterson RA, Kimmel PL: Pain, sleep disturbance, and quality of life in patients with chronic kidney disease. Clin J Am Soc Nephrol 2007, 2:919-925.

26. Hiescu EA, Yeates KE, Holland DC: Quality of sleep in patients with chronic kidney disease. Nephrol Dial Transplant 2004, 19:95-99.

27. Chen SC, Chang JM, Liu WC, Chen YY, Chen LI, Huang JC, Yang TK, Su HM, Chen $\mathrm{HC}$ : The ratio of observed to predicted left ventricular mass is independently associated with increased cardiovascular events in patients with chronic kidney disease. Hypertens Res 2012, 35:832-838

28. Farshid A, Pathak R, Shadbolt B, Arnolda L, Talaulikar G: Diastolic function is a strong predictor of mortality in patients with chronic kidney disease. BMC Nephrol 2013, 14:280.

29. Huang Y, Mai W, Hu Y, Wu Y, Song Y, Qiu R, Dong Y, Kuang J: Poor sleep quality, stress status, and sympathetic nervous system activation in nondipping hypertension. Blood Press Monit 2011, 16:117-123.

30. Tadic MV, Ivanovic BA, Celic VP: Does a nondipping pattern impact the right ventricle in hypertensive patients? Blood Press Monit 2012, 17:47-54.

31. Siddiqi L, Prakken NH, Velthuis BK, Cramer MJ, Oey PL, Boer P, Bots ML, Blankestijn PJ: Sympathetic activity in chronic kidney disease patients is related to left ventricular mass despite antihypertensive treatment. Nephrol Dial Transplant 2010, 25:3272-3277.

32. Harvey AG, Tang NK: (Mis) perception of sleep in insomnia: a puzzle and a resolution. Psychol Bull 2012, 138:77-101.
33. Johansson $P$, Alehagen $U$, Ulander $M$, Svanborg $E$, Dahlstrom $U$, Brostrom A: Sleep disordered breathing in community dwelling elderly: associations with cardiovascular disease, impaired systolic function, and mortality after a six-year follow-up. Sleep Med 2011, 12:748-753.

34. Mellor A, Waters F, Olaithe M, McGowan H, Bucks RS: Sleep and aging: examining the effect of psychological symptoms and risk of sleepdisordered breathing. Behav Sleep Med 2014, 12:222-234.

doi:10.1186/1471-2369-15-131

Cite this article as: Zhang et al:: Association between sleep quality and cardiovascular damage in pre-dialysis patients with chronic kidney disease. BMC Nephrology 2014 15:131.

\section{Submit your next manuscript to BioMed Central and take full advantage of:}

- Convenient online submission

- Thorough peer review

- No space constraints or color figure charges

- Immediate publication on acceptance

- Inclusion in PubMed, CAS, Scopus and Google Scholar

- Research which is freely available for redistribution

Submit your manuscript at www.biomedcentral.com/submit
C) BioMed Central 